Meta

Journal des traducteurs

Translators' Journal

\title{
Pour une mise en valeur de la connaissance historique : une anthologie de textes portugais sur la traduction
}

\section{Manuela Fernández Sánchez et José A. Sabio Pinilla}

Volume 49, numéro 3, septembre 2004

L'histoire de la traduction et la traduction de l'histoire

History of Translation and Translation of History

URI : https://id.erudit.org/iderudit/009385ar

DOI : https://doi.org/10.7202/009385ar

Aller au sommaire du numéro

Éditeur(s)

Les Presses de l'Université de Montréal

ISSN

0026-0452 (imprimé)

1492-1421 (numérique)

Découvrir la revue

Citer cet article

Fernández Sánchez, M. \& Sabio Pinilla, J. A. (2004). Pour une mise en valeur de la connaissance historique : une anthologie de textes portugais sur la traduction. Meta, 49(3), 669-680. https://doi.org/10.7202/009385ar
Résumé de l'article

Suite à de récentes publications espagnoles consacrées intégralement ou en partie à des aspects historiques, nous observons une série de lacunes qui semblent bien loin d'être comblées. Cela est souvent dû à la prétention de faire table rase de la recherche historique antérieure. Le chercheur ne parvient toujours pas à lire le travail de ses collègues, ni à échanger les informations à disposition et cet isolement est lourd de conséquences pour la recherche qui parfois stagne et verse dans la répétition. La publication de travaux pionniers au Portugal ouvre la voie à une meilleure connaissance historique du passé péninsulaire et à une mise en valeur d'un héritage peu connu et trop souvent soumis à des interprétations simplistes. 


\title{
Pour une mise en valeur de la connaissance historique: une anthologie de textes portugais sur la traduction
}

\author{
MANUELA FERNÁNDEZ SÁNCHEZ \\ JOSÉ A. SABIO PINILLA \\ Université de Grenade, Grenade, Espagne \\ mmfs@ugr.es
}

\begin{abstract}
RÉSUMÉ
Suite à de récentes publications espagnoles consacrées intégralement ou en partie à des aspects historiques, nous observons une série de lacunes qui semblent bien loin d'être comblées. Cela est souvent dû à la prétention de faire table rase de la recherche historique antérieure. Le chercheur ne parvient toujours pas à lire le travail de ses collègues, ni à échanger les informations à disposition et cet isolement est lourd de conséquences pour la recherche qui parfois stagne et verse dans la répétition. La publication de travaux pionniers au Portugal ouvre la voie à une meilleure connaissance historique du passé péninsulaire et à une mise en valeur d'un héritage peu connu et trop souvent soumis à des interprétations simplistes.
\end{abstract}

\begin{abstract}
Certain recent publications in Spain devoted partly or wholly to historical matters have brought to light a series of difficulties which are very far from being resolved. The confusion mainly stems from the tendency of many researchers to overlook the research which has already been done in the field. We persist in not reading each other's work, in failing to share information, and the inevitable result of such isolation is repetition and lack of progress. With the publication of pioneer studies from the Portuguese perspective, we can now broaden our knowledge of Peninsular history, and at the same time appreciate the importance of a legacy which is little known and has tended to be oversimplified.
\end{abstract}

\section{MOTS-CLÉS/KEYWORDS}

histoire canonique de la traduction, domaine de la péninsule Ibérique, anthologie de textes portugais sur la traduction, Portugal, théorisation

\section{Introduction}

L'élaboration d'une anthologie de textes théoriques sur la traduction au Portugal (Sabio et Fernández 1998) nous mène à la conclusion que ce n'est pas par manque de dynamisme ou d'intérêt que la traduction portugaise reste inconnue des chercheurs. L'omission du Portugal dans la recherche historique sur la traduction doit être interprétée comme une conséquence directe de la persistance des canons culturels et littéraires. Il semblerait en effet que nous ne nous soyons toujours pas libérés du poids de l'histoire canonique de la traduction qui mentionne certains auteurs, certaines formes de médiation et certaines cultures au détriment du reste, engendrant ainsi l'exclusion de toute une tradition et l'impossibilité pour la recherche d'avancer dans d'autres directions que celles déjà indiquées. 
Cette étude cherche à faire connaître quelques réflexions sur la question de la valeur de la connaissance historique, telles qu'elles ont été élaborées au cours de notre travail sur le discours de la traduction au Portugal entre les $\mathrm{Xv}^{\mathrm{e}}$ et XvinI ${ }^{\mathrm{e}}$ siècles. Cela dit, il ne s'agit pas seulement de diffuser certaines caractéristiques de ce discours ou de faire valoir ses liens avec le contexte européen et péninsulaire, car nous voulons également porter une attention particulière aux questions méthodologiques ayant orienté notre recherche. Par ce travail de réflexion, nous cherchons à démentir les préjugés qui pendant trop longtemps ont accusé l'histoire culturelle portugaise d'être tardive et de manquer d'originalité, conduisant ainsi à des interprétations réductrices d'une réalité très complexe.

\section{Une anthologie de textes théoriques portugais sur la traduction}

Le projet d'une anthologie de textes théoriques portugais sur la traduction est né il y a quelques années d'un travail d'équipe et d'un intérêt particulier pour l'analyse et la diffusion de certains textes clés de l'histoire culturelle portugaise, présentant un travail de réflexion sur la traduction. À la naissance de notre projet, sauf quelques rares exceptions, la pensée et l'activité traductionnelles portugaises restaient inconnues des chercheurs. Quant aux chercheurs portugais eux-mêmes, il s'agissait de philologues et de comparatistes qui s'intéressaient à la traduction, dans le but de mettre en valeur la contribution des textes à l'évolution de la langue et de la littérature portugaises. D'autre part, l’organisation académique des études de traduction au Portugal ne s'est réalisée qu'à la moitié des années 1990. Le mouvement éditorial qui naît de l'institutionnalisation ne s'était donc pas encore produit. D'ailleurs, la première anthologie de textes théoriques sur la traduction, celle de Castilho Pais (1997), est considérée comme un travail pionnier qui réagit dès les premières pages contre l'oubli dans lequel sombraient les traducteurs et la pensée sur la traduction au Portugal (Castilho Pais 1997: 18). Le travail de ce chercheur portugais relança en quelque sorte notre projet de départ car il s'agissait, dès lors, d'apporter une étude complémentaire à ce répertoire de sources réalisé par Castilho Pais. En plus de l'objectif initial déjà exposé, nous avions l'intention d'analyser le discours sur la traduction au Portugal dans une époque déterminée et en rapport avec les conceptions culturelles, linguistiques et littéraires d'un moment donné. Cela impliquait un long travail de recueil de sources et de mise en contexte des textes trouvés, ce qui sans doute allait faciliter par la suite la reconstitution historique des différentes pratiques textuelles, entre autres les traductions (Fernández et Sabio 1998).

Il s'agissait de réunir, dans la première étape de notre recherche, un important corpus de textes, suffisamment varié et représentatif du discours sur la traduction au Portugal entre les $\mathrm{XV}^{\mathrm{e}}$ et $\mathrm{XVIII}{ }^{\mathrm{e}}$ siècles. Nous avons limité notre recherche à cette période $\mathrm{du}$ fait de l'importance décisive qu'elle a pu jouer dans la configuration du système linguistique, littéraire et culturel. Mais c'est aussi parce qu'à plus grande échelle ce cadre spatio-temporel, considéré dans son contexte européen occidental, nous permettait d'utiliser tout d'abord la tradition unitaire latino-chrétienne comme point de repère, et puis, également, l'influence castillane et l'influence française.

La recherche historique que nous voulions réaliser passait par plusieurs étapes que D'Hulst (1995) a systématisées. Nous ne nous y attarderons pas ici car elles sont commentées de façon détaillée dans notre anthologie. 
Au cours de cette analyse, nous porterons une attention particulière aux différentes formes et fonctions de la réflexion théorique sur la traduction et nous aurons recours à un nombre restreint d'exemples qui ont été sélectionnés en fonction de leur caractère singulier et représentatif de la réflexion portugaise sur la traduction. Enfin, avant de rentrer dans le vif du sujet, il faut ajouter que nous sommes conscients des risques que comporte une telle tâche d'analyse, surtout quand nous ne disposons pas en général de recherches consacrées au domaine portugais qui soient basées sur des corpus de textes suffisamment larges ${ }^{1}$.

\section{Analyse du discours sur la traduction au Portugal du XV $\mathrm{XV}^{\mathrm{e}} \mathrm{au} \mathrm{XIII}^{\mathrm{e}}$ siècles}

Au cours de l'élaboration du corpus et à la suite d'un long travail de recueil de sources les plus diversifiées possibles, nous nous sommes vite rendu compte que les textes appartenaient à des genres différents, ce qui devait être pris en compte pour bien comprendre les conditions de leur fonctionnement historique. Nous avons donc organisé les 44 textes sélectionnés en trois catégories (préliminaires, normatifs et critiques) suivant dans chaque catégorie un ordre chronologique. Il ne faut pas s'étonner du nombre inégal de textes entre les catégories (textes préliminaires: 34 , textes normatifs: 17, textes critiques: 3 ). Cela reflète tout simplement ce que nous avons pu trouver. Nous aurions même pu augmenter le nombre de textes préliminaires, étant donné que ce sont les plus nombreux dans la période étudiée.

Étant donné la diversité de formes discursives, une mise en contexte des documents étudiés a été fondamentale pour éviter, dans la mesure du possible, de passer outre certains détails importants qui à première vue auraient pu sembler insignifiants. Nous y ferons référence plus loin.

\subsection{Textes préliminaires}

Nombreux sont les chercheurs qui ont tenté d'attirer l'attention sur les problèmes d'interprétation des commentaires sur la traduction qui figurent dans les dédicaces, les prologues et les préfaces. Dans le cadre de notre recherche, une lecture attentive de ces textes confirme la valeur justificative et défensive, en plus de sa valeur rhétorique, de la réflexion sur la traduction, qui dans la période que nous analysons est particulièrement liée aux différentes formes et fonctions, adoptées à chaque époque et dans chaque situation sociale, dans le but d'accéder à la connaissance des textes littéraires.

Mais c'est aussi parce qu'il s'agit d'un espace privilégié où prend place la communication entre le traducteur et le lecteur que les textes préliminaires nous ont semblé importants. C'est effectivement un espace qui fournit des informations précieuses sur les attentes du lecteur de la traduction à une époque déterminée, ainsi que sur les circonstances dans lesquelles le traducteur entreprend sa tâche. C'est peut-être l'information la plus digne de foi qui puisse être trouvée dans des textes de nature essentiellement rhétorique. De plus, il s'agit de réflexions venant des traducteurs eux-mêmes, pour la plupart des écrivains, des poètes ou des professeurs. 


\subsubsection{La valeur justificative de la théorisation}

Le contexte de la tradition latino-chrétienne, qui est notre point de départ, laisse place au discours sur la traduction avec une fonction éducative dominante et voit naître dans la cour portugaise au début du $\mathrm{Xv}^{\mathrm{e}}$ siècle, parallèlement à ce qui se passait dans les cours européennes, un courant de divulgation de la culture classique; et cela, grâce au soutien que l'Infant D. Pedro (1392-1448) et son frère le roi D. Duarte (1391-1438) ont porté aux traductions et aux compilations, dans un moment qui correspond à la naissance de la langue portugaise comme langue littéraire. S’il y a quelque chose qui attire particulièrement l'attention du chercheur qui analyse les prologues des œuvres de l'Infant D. Pedro, compilateur de Sénèque et traducteur de Cicéron, c'est bien la finesse et l'intérêt avec lesquels il s'est arrêté sur les aspects linguistiques et organisationnels du discours, propres au processus de manipulation et d'élaboration du matériel associé à la pratique de la traduction; mais encore la cohérence et l'originalité avec lesquelles il a entrepris sa tâche, démontrant ainsi la valeur et l'utilité de la communication en langue vulgaire.

Le plus grand jurisconsulte et chroniqueur du royaume, Vasco Fernandes de Lucena (? - 1499?), est un autre élément exceptionnel pour le chercheur en histoire de la traduction dans le domaine de la péninsule Ibérique. En effet, celui-ci représente au Portugal, comme son parent Vasque de Lucène à la cour de Bourgogne, le traducteur au service de l'idéal chrétien cautionné par le pouvoir politique. Il est également l'héritier par excellence de l'exigence de fidélité à la pensée de l'auteur, notion imposée par la tradition latine transmise par saint Jérôme, mais qui semble chez Vasco Fernandes de Lucena être plus élaborée et plus appliquée à son travail. Voyons, par exemple, quelques lignes de son prologue à la traduction du traité De Senectute de Cicéron (élaborée entre 1438 et 1448):

[...] empero porque [v] os praz de lerdes por livros de linguagem, por aproveitardes a muitos me trabalhei de o trasladar, não porque o livro seja mais doce, mas porque seja mais comum, ca certamente o pequeno bem milhor é que o bem singular, e em isto não tirei letra de letra, que seria trasladar, nem sentença de letra, que seria glosar, mas tirei sentença de sentença, que é bem e proveitosamente interpretar [...] (Sabio et Fernández $1998: 50)^{2}$.

Il faut relever par exemple la référence aux différentes manières de traduire qui vise à une absence de critères établis en ce qui concerne l'activité de la traduction, et il faut observer surtout l'option de traduction qu'il propose, partagée par la majeure partie des traducteurs de la Péninsule du $\mathrm{Xv}^{\mathrm{e}}$ siècle, et qui peut être résumée en une expression connue qui fait allusion au transfert rigoureux de la substance: «tirar sentença de sentença».

D’autres idées exposées dans ce prologue et propres au contexte péninsulaire du $\mathrm{XV}^{\mathrm{e}}$ siècle font référence au topique de l'infériorité de la langue vulgaire par rapport à la langue latine, préjugé qui restera ancré au moins jusqu'au $\mathrm{XvI}^{\mathrm{e}}$ siècle.

\subsubsection{Un ensemble d'idées sur la traduction disponibles dans la péninsule Ibérique}

La référence au travail réalisé par Valentim Fernandes dans son atelier de Lisbonne entre 1495 et 1518 est incontournable. À la fin du $\mathrm{Xv}^{\mathrm{e}}$ siècle, en pleine époque de l'introduction de l'imprimerie au Portugal, celui-ci joua un rôle fondamental en garantissant la continuité des modèles littéraires et religieux des siècles antérieurs. Il 
faut mentionner l'impression en 1495 de la Vita Christi, l'un des livres les plus influents à cette époque, et l'élan donné à de nouveaux genres qui voyaient souvent le jour sous la forme de traduction en langue vulgaire (romans de chevalerie et compilations sur l'expansion maritime).

Nous avions déjà été interpellés, lors de l'analyse du personnage complexe et insaisissable de Valentim Fernandes, par l'importance qu'il attachait à l'impression de livres en langue vulgaire et la familiarité avec laquelle il employait les topiques de valeur et d'utilité en rapport avec cette activité. Mais c'est surtout la réflexion théorique présentée dans le prologue du Livro de Nicolao Veneto, faisant partie intégrante de son œuvre Marco Paulo (1502), qui selon nous revêt une importance particulière. En effet, cette réflexion vient corroborer une hypothèse que nous avions déjà avancée au cours de travaux antérieurs (Sabio et Fernández 2001) et qui laisserait envisager l'existence d'un ensemble d'idées sur la traduction plus ou moins disponibles dans une bonne partie de la Péninsule.

Le passage que nous voulons commenter ici a été présenté par des chercheurs comme une pièce curieuse de méditation philosophique et de rhétorique littéraire et comme une preuve de la maîtrise de la langue portugaise à laquelle était parvenu Valentim Fernandes (Anselmo 1981: 174; Anselmo 1987: 30). Toutefois, personne n'a encore à ce jour souligné qu'il s'agit en grande partie d'une traduction du prologue que l'humaniste castillan Alfonso de Palencia avait écrit en 1457 pour la Batalla campal de los perros contra los lobos. Il est intéressant de comparer le fragment du prologue de Valentim Fernandes avec celui qui apparaît dans le prologue d'Alfonso de Palencia:

Ainda que se me faz mui grave o trasladar de latim em linguagem, conhecendo os defeitos que assim em o som das cláusulas como em a verdadeira significação de muitos vocábulos que de necessário vêm às trasladações de uma língua em outra, convém de falar por circunlocuções ou rodeios (Sabio et Fernández 1998: 54) ${ }^{3}$.

Et como quiera que mucho se me faga grave el romanzar, sabiendo las faltas que así en el són de las cláusulas como en la verdadera significación de muchos vocablos, de necesario vienen en las translaciones de una lengua a otra, mayormente en lo que de latín á nuestro corto fablar se convierte (Sabio et Fernández 2001: 99)

Il faut reconnaître que le choix de l'imprimeur d'utiliser Alfonso de Palencia est surprenant, étant donné que ce dernier est un humaniste castillan qui s'érige en ennemi des traductions et qui remet en question la possibilité de traduire de façon efficace du latin à la langue vulgaire sans se contenter exclusivement du transfert du contenu. En tout cas, nous pouvons sans hésiter émettre l'hypothèse que Valentim Fernandes connaissait cette œuvre, qu'il appréciait la prose élégante et équilibrée de ce castillan instruit et qu'il se soit servi de ce passage sur les difficultés de la traduction, élaboré par Palencia, pour prononcer son propre discours et reformuler à sa façon les conventions attendues dans ce type de textes préliminaires.

Ce texte, ainsi que d'autres du même imprimeur, nous permet de mieux comprendre le discours sur la traduction de cette époque associé à une pratique justificative des traductions en langue vulgaire, pratique commune au domaine péninsulaire et européen. Il fournit aussi des informations sur la manière dont les hommes instruits de l'époque pouvaient avoir recours dans l'exercice de cette pratique à des idées plus ou moins élaborées et connues sur le discours autour de la traduction dans la péninsule Ibérique. 


\subsubsection{La valeur défensive de la théorisation}

L'influence castillane est constante et atteint son apogée au cours des $\mathrm{XVI}^{\mathrm{e}}$ et $\mathrm{XVII}^{\mathrm{e}}$ siècles. Cette époque se distingue donc sur le plan culturel et politique par la forte identité linguistique et littéraire et les traits dynastiques communs au Portugal et à l'Espagne, facteurs qui ne se reproduiront plus à l'avenir. Quelques exemples seront suffisants pour en montrer les répercussions sur l'activité et sur la pensée traductionnelles, dans un moment historique marqué par le bilinguisme des classes cultivées et par la suprématie du castillan au-delà de ses frontières.

Pedro Nunes (1502-1578), un humaniste mathématicien et cosmographe du royaume, précepteur des infants et professeur à l'université de Coïmbre, réalisa en 1564 la traduction en castillan d'une ouvre qu'il avait lui-même écrite en portugais vers 1534, le Libro de álgebra. Dans la dédicace, il est facile de percevoir la valeur défensive de la théorisation liée à la traduction en portugais, trait caractéristique de la réflexion sur la traduction à cette époque:

E primeiramente a escrevi em nossa língua Portuguesa, e assim a viu V. A., mas depois considerando que o bem quanto mais comum e universal, tanto é mais excelente, e porque a língua Castelhana é mais comum em toda a Espanha que a nossa, por esta causa a quis trasladar em língua Castelhana, para nela se haver de imprimir, porque não careça dela aquela nação tanto nossa vizinha com a qual tanto comunicamos, e tanta amizade temos (Sabio et Fernández 1998: 65) ${ }^{5}$.

$\mathrm{Au} \mathrm{XvI} \mathrm{e}^{\mathrm{e}}$ siècle, les écrivains portugais n'écrivaient pas seulement en castillan mais rédigeaient également des versions castillanes du latin, du français et de l'italien et même parfois du portugais comme Pedro Nunes. Seulement quatre écrivains portugais du XVI ${ }^{e}$ siècle n'écrivirent pas en castillan: Bernardim Ribeiro, António Ferreira, frère Agostinho da Cruz et Jorge Ferreira de Vasconcelos. Quant aux autres, de Gil Vicente à Camões, ils n'hésitèrent pas à utiliser le castillan comme option stylistique. La suprématie de la langue poétique des cancioneiros galiciens et portugais au Moyen Âge céda le pas devant le castillan, plus présent au cours du $\mathrm{Xv}^{\mathrm{e}}$ siècle, au point que le castillan soit utilisé par $70 \%$ des poètes dans le Cancioneiro Geral de Garcia de Resende (1516). C'est la prédominance et le prestige du castillan qui favorisent le rôle de système source de la littérature castillane. En effet, l'expansion impériale de la langue fait du castillan une langue diplomatique ainsi qu'un instrument de communication à niveau administratif et parmi les élites de la Péninsule. Le castillan devient si familier à la fin du $\mathrm{XvI}^{\mathrm{e}}$ siècle que Simão Lopes, traducteur de Flos sanctorum d'Alonso de Villegas en portugais, formule le commentaire suivant en 1598: «Não há hoje em Portugal pessoa a quem os livros castelhanos lhe não sejam tão naturais como os próprios portugueses» (Viterbo s. d.: 117). Cette familiarité du castillan grandira encore pendant la période de la double monarchie (1581-1640) comme le montre avec une foule de détails Domingo Garcia Perez dans son catalogue (1890).

Toutefois, nous ne pouvons ignorer d'autres témoignages de la même époque qui en apparence semblent contradictoires, et qui défendent l'utilité des traductions en portugais d'œuvres appréciées pour leur aspect didactique, comme celles de Cicéron par exemple. Ainsi, l'un des traducteurs les plus importants à l'époque, Duarte de Resende, en 1531 défend avec fièvre la langue portugaise comme langue littéraire dans la «carta» qui précède sa traduction des traités de Cicéron, prenant ainsi quel- 
ques années d'avance sur les grammaires de Fernão de Oliveira (1536) et de João de Barros (1540) qui donneront à ce sujet toute l'importance qu'il mérite.

\subsubsection{La réflexion sur la traduction dans les traductions d'œuvres poétiques}

L'influence du castillan coïncide avec l'essor autonome du portugais comme langue littéraire; cela ayant des répercussions négatives sur la pratique et sur la pensée traductionnelles. Si nous consultons dans le premier volume de l'œuvre de Gonçalves Rodrigues (1992) la période que nous étudions, nous pouvons observer que les $\mathrm{XVI}^{\mathrm{e}}$ et $\mathrm{XVII}^{\mathrm{e}}$ siècles se trouvent parmi les plus pauvres en traduction portugaise, en nombre et diversité d'auteurs. La plupart des traductions sont des œuvres spirituelles écrites en latin ou en castillan; les deux langues qui vont avoir le plus d'influence sur le portugais au moins jusqu'au Xvin' ${ }^{\mathrm{e}}$ siècle.

Si nous observons le XVII ${ }^{\mathrm{e}}$ siècle portugais, le poème épique Os Lusíadas (1572) constitue une référence incontournable à la littérature et par conséquent à la traduction. Les traductions en castillan, les éditions commentées ainsi que les polémiques autour de l'épopée de Camões avivèrent l'intérêt pour la poésie épique au XVII ${ }^{\mathrm{e}}$ siècle. Et c'est précisément dans les traductions poétiques, concrètement les traductions des œuvres de Virgile et du Tasse, ainsi que dans la critique de la poésie épique, que nous trouverons d'autres témoignages intéressants pour notre travail.

Dans ce contexte, il faut mentionner Leonel da Costa (1570-1647), premier traducteur d'une ouvre complète de Virgile en portugais, les Éclogas e Geórgicas (1624), avec une finalité didactique qui le rapproche de l'attitude des grammairiens comme Amaro de Roboredo. Le but recherché était que les élèves apprennent à bien construire le latin à partir de la version et sur la base des commentaires proposés. L'intention de Leonel da Costa est en ce sens complètement opposée à celle de João Franco Barreto (1600-1674 ?). Ce dernier, qui réalisa la traduction de l'Énéide en ottava rima, eut recours au modèle littéraire de l'épique, ce qu'il faut interpréter comme une nouvelle approche de la traduction comme activité littéraire et comme une alternative aux traductions scolaires des classiques, à la manière de Leonel da Costa, auteur d'une traduction manuscrite de l'Énéide. En ce qui concerne la réflexion sur la traduction, l'allusion de Franco Barreto à son désir de plaire au lecteur est à souligner car, comme le montre bien Castilho Pais (1997: 33), elle ouvre la voie à une plus grande liberté du traducteur:

No que pus toda minha diligência foi em não me apartar do sentido do Poeta, conservando inteiras suas sentenças, tropos e figuras, e em fazer doce e sonoro o verbo, para dar gosto ao Leitor, que traduzir palavra por palavra, nem ainda em prosa se pode bem fazer, e quem inviolavelmente o quiser observar deitará tudo a perder, que por essa razão Luís Vives chama imperitos aos que assim traduziram as obras de Aristóteles (Sabio et Fernández 1998: 74) ${ }^{6}$.

Enfin, nous pouvons observer dans les prologues de Leonel da Costa et de Franco Barreto des idées en commun: le manque de traductions en portugais, ce qui vient justifier l'appel patriotique des traducteurs et le sujet constamment présent de la traduction de l'hexamètre latin ainsi que de la construction du vers en langue vulgaire. Leonel da Costa opte pour le vers libre alors que Franco Barreto choisit l'ottava rima. Le personnage de Camões est au cœur du débat, ce qui n'est pas surprenant dans un siècle marqué par les polémiques autour du statut de modèle épique d'Os Lusíadas. 
Mais penchons-nous maintenant sur le personnage d'André Rodrigues de Matos (1638-1698), traducteur de Jérusalem délivrée du Tasse en ottava rima en 1682. Dans le prologue de la traduction, nous pouvons relever des commentaires communs aux nombreux prologues du XVIII ${ }^{\mathrm{e}}$ siècle: la conception de la traduction comme une copie de l'original, le manque de considération du traducteur, les difficultés de la traduction et la capacité de la langue portugaise à traduire le latin et d'autres langues vulgaires. D’autre part, ces idées justifient l'utilité des traductions, défendent la méthode utilisée et font preuve de la confiance accordée à la valeur littéraire de la langue portugaise. L'ambiguïté qui caractérise le discours portugais sur la traduction à cette époque est en grande partie une influence du discours français lui-même, ainsi qu'un témoignage de la situation précaire des traducteurs portugais face à une définition de la traduction qui oscille entre un exercice de style et une activité soumise à des normes.

\subsection{Textes normatifs}

Dans cette catégorie, nous recueillons d'autres formes discursives qui, présentant une singularité ou constituant une référence pour leur époque, sont d'excellents outils pour notre recherche sur la situation historique de la traduction. Jusqu'au $\mathrm{xx}^{\mathrm{e}}$ siècle, tous les essais théoriques systématiques provenant de la péninsule Ibérique sont subordonnés à une pratique de la traduction. Juan Luis Vives qui en 1532 dans De arte dicendi consacre déjà un chapitre à la réflexion théorique sur la traduction, semble être une exception à la règle.

Dans cette partie de l'anthologie, les réflexions sur la traduction recueillies proviennent de commentaires qui apparaissent tout au long d'une œuvre, comme c'est le cas du chapitre 99 du Leal Conselheiro du roi D. Duarte, élaboré entre 1437 et 1438. Cependant, cela ne veut pas dire que les conseils du roi pour bien traduire le latin eussent été insignifiants. Bien au contraire, ils ont un siècle d'avance sur la réflexion théorique d'Étienne Dolet et font part de la préoccupation de ce roi illustre quant à l'inexistence de critères de traduction tout en montrant son intérêt personnel à systématiser cette pratique.

Nous incluons également dans la catégorie des textes normatifs des réflexions occasionnelles apparaissant dans les descriptions grammaticales des premiers grammairiens portugais d'Oliveira et de Barros et apparaissant dans le cadre de l'étude comparative des langues du grammairien et pédagogue Amaro de Roboredo. De façon analogue, nous incluons dans les textes normatifs l'entrée des mots «tradução» et «traduzir» mentionnés dans l'œuvre encyclopédique de Bluteau (Vocabulário Português e Latino, 1710-1728), et enfin nous relevons le message d'alerte lancé par l'illustré Luís António Verney (1713-1798) contre l'inertie des méthodes d'enseignement dans les matières grammaticales (Verdadeiro Método de Estudar, 1746).

D’une façon générale, puisqu'il y a de grandes différences parmi eux, ce sont des textes normatifs, pensés à des fins éducatives et didactiques, qui se sont définies au fil de l'histoire culturelle européenne, et qui trouvent leur correspondance au cœur du renouveau intellectuel péninsulaire dont Alphonse X le Sage est à l'origine; au cour du mouvement de défense et de valorisation des langues vulgaires; lors des premières grammaires rationalistes sous l'influence de El Brocense; lors des premières œuvres lexicographiques et enfin au cour du renouveau pédagogique, devant lequel l'enseignement traditionnel représenté par les Jésuites cède le pas. 


\subsubsection{La traduction dans l'enseignement des langues}

Les défenseurs de la langue portugaise, non seulement au $\mathrm{XvI}^{\mathrm{e}}$ siècle mais aussi et surtout au XVII ${ }^{\mathrm{e}}$ siècle, ont su réagir face aux méthodes d'enseignement scolaire de la langue vulgaire qui n'apprenaient aux enfants qu'à lire et à écrire sans aucune base de grammaire.

Ce sont des pédagogues comme Roboredo qui vont se démarquer des méthodes grammaticales traditionnelles, et des illustres comme Verney, ayant voyagé et découvert de nouvelles doctrines linguistiques et de nouvelles méthodes d'apprentissage, qui vont accuser l'enseignement du latin dans les écoles d'immobilisme et d'inertie sur les questions grammaticales.

Par rapport aux grammaires normatives en langue vulgaire, l'œuvre de Roboredo, son Método Gramatical (1619), est un exemple de grammaire générale et universelle inspirée de El Brocense qui maintient une position rationaliste, basée sur l'égalité de la raison parmi les êtres humains et, par conséquent, sur la possibilité de dicter des normes communes à toutes les langues. Il faut souligner que dans l'œuvre de Roboredo, la langue maternelle, le portugais, sert d'outil de description du latin. L'idée qu'une méthode didactique puisse être appliquée à n'importe quelle langue ou puisse servir à étudier les langues des terres découvertes et à enseigner aux peuples lointains la langue portugaise est particulièrement intéressante.

Quant au discours sur la traduction, de nombreuses références apparaissent dans l'œuvre de Roboredo quand le pédagogue explique les exercices que l'élève doit réaliser dans le processus d'apprentissage des langues. La traduction est présentée comme un outil d'enseignement et de description des langues, orienté vers l'observation des différences structurales et stylistiques existantes entre la langue maternelle et les langues étrangères.

\subsection{Textes critiques}

Dans le cas des textes normatifs, la réflexion sur la traduction est subordonnée à l'enseignement des langues et va de pair avec la mission universaliste d'une époque exceptionnelle, comme celle de l'expansion maritime portugaise. Pour les textes critiques, la réflexion sur la traduction est présentée autrement: dans le premier texte, celui de Manuel Pires de Almeida, la réflexion sur la traduction apparaît sous la forme de notes critiques sur la traduction poétique ( $D a$ Tradução dos Poetas, élaboré entre 1634 et 1640); dans le deuxième, celui de Correia Garção, elle semble être au service d'une doctrine esthétique qui aspire à l'imitation créatrice des auteurs portugais classiques et du XVI ${ }^{\mathrm{e}}$ siècle (Dissertação Terceira: Sobre o prinicipal preceito para formar um bom poeta, 1757); enfin, dans le troisième texte, elle apparaît dans un traité de traduction qui cherche à réguler l'activité littéraire selon le bon goût: chapitre I de Arte de traduzir de latim para português (1818) de Sebastião José Guedes et d'Albuquerque.

À part le texte de Correia, les deux autres sont des traductions de théoriciens français, Du Bellay et Philipon de la Madelaine respectivement. La liste de plagiaires portugais ou d'influences plus ou moins serviles exercées sur les auteurs portugais par des auteurs d'œuvres poétiques ou de traités d'éloquence français, castillans et italiens est longue et c'est un fait qui montre l'influence de la critique et de l'esthétique française, surtout en ce qui concerne l'élaboration d'une doctrine néoclassique portugaise. 
Un exemple qui illustre bien l'influence française est le traité de traduction d'Albuquerque dont la source non avouée est le traité de Louis Philipon de la Madelaine (1762): L'art de traduire le latin en français, réduit en principes, à l'usage des jeunes gens qui étudient cette langue.

Tout d'abord il faut signaler qu'Albuquerque dans la préface situe son travail dans le domaine des Belles-Lettres, non pas dans le domaine grammatical, étant donné que son objectif est d'enseigner le bon goût. Albuquerque admet à plusieurs reprises qu'il n'en est pas à l'origine. Suivant son prédécesseur, il affirme que son œuvre vient palier un manque du fait de n'avoir trouvé aucun traité en portugais qui facilite la tâche aux jeunes gens de copier les modèles anciens. De façon analogue à L. P. de la Madelaine, qui lui-même fait référence à des voix contemporaines autorisées comme Rollin et Batteux, Albuquerque prétend réduire à des principes un art qui ne propose des règles que trop générales. Les six premiers chapitres sont consacrés aux aspects principaux de la traduction et au génie des langues; le chapitre 7 est consacré aux règles générales de la traduction: bien connaître la valeur de chaque terme et tout ce qui est en rapport avec la phrase; aspects hérités de la tradition grammaticale (D’Hhulst 1996: 93). Le chapitre 8, un peu plus long, est consacré aux règles spécifiques de la traduction en rapport avec la rhétorique comme les figures de style, l'ordre des différents éléments de la phrase et les façons de parler propres au latin. C'est peut-être la partie finale sur les modèles de traduction pour débutants qui est la plus intéressante du fait de son originalité. Des travaux de traducteurs portugais contemporains sont présentés comme modèles de traduction et des notes, qui sont le reflet de tout un exercice critique réalisé par Albuquerque sur la manière de traduire des différents traducteurs, y sont ajoutées.

Quant au rôle joué par la traduction dans ce traité, il semble servir de soutien aux textes latins qui sont étudiés comme modèles. Ce rôle n'est pas clairement défini vu qu'il oscille entre la grammaire et l'idéal esthétique du bon goût propre à cette époque.

\section{Conclusions}

Malgré les limites de ce travail, nous espérons avoir démontré que le fonctionnement de la réflexion théorique sur la traduction, dans un espace culturel et une période historique donnés, ne peut être évalué sans considérer le rapport existant entre les différentes formes discursives et les pratiques sociales. La valeur défensive de la théorisation, par exemple, peut rester dans l'ombre si nous limitons notre recherche aux contenus, sans tenir compte de l'ensemble des relations posées par les textes et qui nous renvoient à un contexte plus large dans lequel elles trouvent certainement leur explication. À la différence d'autres anthologies du même type, souvent consacrées à la diffusion des textes les plus représentatifs sur la traduction à une époque donnée, notre travail réunit un grand nombre de textes très différents les uns des autres, du point de vue de leur thématique et de leur influence dans la culture de l'époque considérée. Notre parcours historique à travers le discours portugais sur la traduction nous aura appris non seulement à identifier de nouvelles données comme la réflexion sur la traduction lyrique et épique ou l'influence française au xvIII siècle, mais encore à nuancer ou remettre en question bien d'autres informations comme la revendication d'un contexte péninsulaire dans la recherche historique sur la traduction au Portugal. Et cela n'a rien de superficiel dans le contexte péninsulaire où nous 
réalisons notre recherche. Comme nous l'avons dit au début de ce travail, il n'y a pas eu de renouveau significatif de la connaissance du passé. De ce fait, c'est encore l'histoire canonique de la traduction péninsulaire qui sert de référence. D'ailleurs, dans les derniers ouvrages d'orientation générale publiés en Espagne, la recherche historique qui a été réalisée sur la tradition portugaise ces dernières années n'est même pas mentionnée. Nous pensons avoir apporté des preuves suffisantes pour qu'il soit difficile aujourd'hui de justifier l'exclusion portugaise. Voilà la valeur que nous attribuons à la connaissance historique sur la traduction: c'est sa capacité à devenir un outil de recherche précieux pour donner aux contributions du passé leur juste valeur et éviter à tout moment des interprétations radicales, inexactes et statiques.

\section{NOTES}

1. L'excellente bibliographie de Gonçalves Rodrigues (1992) en est l'exception. Il s'agit d'un répertoire de sources extraordinaire qui, sans être exhaustif, comme le confesse l'auteur lui-même, nous a été d'une grande aide en nous renvoyant à d'autres répertoires bibliographiques. D'autre part, Rener (1989: 6) disait la chose suivante: «In addition, the major portion of the material collected by previous scholarly research in all major languages of Western Europe has been consulted. If some languages have not been as active as their French, English an German colleagues. Portuguese and Slavic languages have even less to offer as far as available information on primary sources is concerned.»

2. [...] mais parce que vous prenez plaisir à lire des livres en langue vulgaire, parce que cela serait d'une grande utilité pour beaucoup, je me suis mis à la tâche de le traduire, et non pour rendre le livre plus doux, mais afin qu'il soit plus commun, car en effet ce qui est petit est bien meilleur que ce qui est singulier, et pour ce faire je ne l'ai pas traduit lettre par lettre, conformément à ce qu'on entend par 'trasladar', ni par des sentences, conformément à ce qu'on entend par 'glosar', mais je l'ai traduit pensée par pensée, qui est bien et profitablement 'interpretar' [...]

3. Et puisque je trouve assez lourd de traduire le latin en langue vulgaire, étant donné les défauts à propos de la sonorité des phrases et de la signification réelle de beaucoup de mots qui accompagnent obligatoirement les traductions entre les langues, il convient de parler au moyen de circonlocutions et de détours.

4. Et puisque je trouve assez lourd le fait de traduire en langue vulgaire, étant donné les erreurs quant à la sonorité des phrases et la signification réelle de beaucoup de mots, qui accompagnent les traductions entre les langues, notamment la langue latine par rapport à l'étroitesse de notre langue.

5. Et tout au début je l'ai écrit dans notre langue portugaise, et c'est ainsi que Votre Altesse a pu le voir, mais depuis que j'ai considéré que le bien a d'autant plus de mérite qu'il est plus commun et universel, et parce que la langue castillane est plus commune que la nôtre partout en Espagne; c'est pour cela que je l'ai traduit en castillan, et c'est dans cette langue que la traduction sera publiée, afin que cette nation ne se voit privée d'elle, une nation qui est notre voisine et avec laquelle nous sommes toujours en communication et liés par une grande amitié.

6. J'ai tenu à ne pas m'éloigner du sens du Poète, j'ai maintenu absolument ses phrases, ses tropes et ses figures, et pour faire plaisir au lecteur j'ai rendu plus douce et plus sonore ma parole, et je n'ai pas traduit mot à mot, ce qui ne peut jamais bien se faire, même pas en prose; et qui mène inévitablement celui qui le pratique à tout risquer, et c'est pour cela que Luis Vives a qualifié d'inexpérimentés les traducteurs d'Aristote qui ont traduit de cette façon ses œuvres.

\section{RÉFÉRENCES}

Anselmo, A. (1981) : As Origens da Imprensa em Portugal. Lisbonne: Imprensa Nacional-Casa da Moeda.

— (1987) : «Valentim Fernandes ou a mediação na alteridade», Revista da Biblioteca Nacional, Série 2 2-2, 27-32.

Castilno Pais, C. (1997): Teoria Diacrónica da Tradução Portuguesa. Antologia (Séc. XV-XX), Lisbonne, Universidade Aberta. 
D’Hulst, L. (1995): «Pour une historiographie des théories de la traduction: questions de méthode», TTR 8-1, 13-33.

— (1996): "Unité et diversité de la réflexion traductologique en France (1722-1789), in M. Ballard et L. D'Hulst, La traduction en France à l'âge classique, Villeneuve d'Ascq (Nord), Presses universitaires du Septentrion, 83-100.

Fernández Sánchez, M. et J. A. Sabio Pinilla (1998) : "Historiografía y traducción: a propósito de una antología de textos teóricos portugueses sobre traducción (ca. 14291818) », Communication présentée au Second International Congress of European Society for Translation Studies (EST), 23-26 septembre, Grenade.

Garcia Peres, D. (1890): Catálogo biográfico y bibliográfico de los autores portugueses que escribieron en castellano, Madrid.

Gonçalves Rodrigues, A. A. (1992): A Tradução em Portugal. Vol. I. 1495-1834. Lisbonne, Imprensa Nacional-Casa da Moeda.

Rener, F. M. (1989): Interpretatio. Language and Translation from Cicero to Tytler, Amsterdam/ Atlanta, Rodopi.

Sabio Pinilla, J. A. et M. Fernández Sánchez (1998): O discurso sobre a tradução em Portugal. O proveito, o ensino e a crítica. Antologia (c. 1429-1818). Lisbonne, Colibri.

Sabio Pinilla, J. A. et M. Fernández Sánchez (2001): "O Marco Paulo de Valentim Fernandes: uma contribuição singular para a história da tradução peninsular ", Discursos 1, p. 87-102.

Viterbo, S. (s. d.): A Literatura Hespanhola em Portugal, in História e Memórias da Academia, Lisbonne, tome II, part II, no 5. 